\title{
Study on the Optimization Method of Transducer Linear Transmission Function Based on Structural Parameter Adjustment
}

\author{
Guangxu Lin \\ Chengdu University, Chengdu 610106, China \\ E-mail: 670149343@qq.com
}

\begin{abstract}
The basic task of the transducer is to convert non-electric physical quantity into electric physical quantity, and implement effective linearization processing to the acquired physical quantity and offer ideal data chain for later measurement circuit. The single variable transmission character of transducer is the quality attributes function about above tasks, which reflects the corresponding relation between input and output, i.e. the linearization degree between both physical quantities. Taking a sort of capacitance transducer as the sample, in this article, we put forward a sort of exact transmission character optimization method of single variable linear correlation analysis with strong operation feature based on the base of statistics. According to this method, for each sort of transducer, the correlation coefficient between output and input can be conveniently computed, and the linearization quality of transducer can be evaluated according to the correlation coefficients, and finally the transducer can achieve ideal linearization level through the adjustment of structure parameter, which can offer sufficient optimization space for the quantity transmission character and manufacturing techniques.
\end{abstract}

Keywords: Quantity transmission character, Optimization method, Linear correlation analysis, Correlation coefficient

\section{Introduction}

In the domain of autoimmunization detection technology, the transmission function between output and input of transducer is decided by not only the basic work principle of physics, but the concrete structure of transducer in the technical design, and the quality of the acquired transmission function is related with the correlation between output and input. For changes in the world, the change of one phenomenon always depends on the change of other phenomena, which is the correlation character among things. In theory, for any one transducer, its transmission function of physical quantity will always present obvious linear correlation character in certain value region, and it can be reflected by diagram or mathematical relation, and if the value region range contained by the linear correlation of this physical quantity is just consistent with the objective value region of the transducer, so it is the ideal situation. But in fact, both parties always are not completely consistent, or they are just partly consistent, i.e. in the value region what we needs, the transmission function is just linear in part of region, and in other parts of region, the correlation among physical quantities always presents complex nonlinear relationship, which will certainly brings difficulties for the later processing works after data acquisition, and if we can exactly grasp the quantity transmission character of the transducer in the phase of structure design, and make correlation evaluation ever and again, we can adjust the quantity transmission character in certain range through the structure adjustment, and implement effective prophase optimization for the latter application. 


\section{Quantity transmission character and representation form of transducer}

In the physical world, the mutual relationships among natural phenomena are very complex, and they are restricted and influenced each other by different orientations, values and degrees. Concretely speaking, the attribute tokens of natural phenomena are mixed physical quantities, and in the development work of transducer, we should evaluate the correlation relationship between input and output, and primarily confirm whether there are obvious correlation relationships between them, and if rough linear correlation exists between them, we can find out the structure parameters which obviously influence the transmission function, and implement corresponding quantitative analysis. According to each data inputting into the function, we should make corresponding correlation diagram and correlation table, then we can directly judge the form of the transmission function and compute the correlation coefficient to implement quantitative analysis, which can exactly reflect their correlation relationships, and these correlation relationships are the quantity transmission character what we said. Generally speaking, the quantity transmission character of the transducer can be represented as two forms, i.e. the scatter diagram method and the correlation coefficient method.

\subsection{Scatter diagram method}

According to the acquired transducer input and output data table, take the measured physical quantity as the transverse axis and the output quantity as the longitudinal axis, label the data group one by one on the diagram, and the corresponding scatter diagram can be obtained. The scatter diagram method can clearly denote the correlation character between input and output of transducer (seen in Figure 1), but its precision is not high, and especially for the transducer which requires high linearization, this method can not be used to establish exact quantitative analysis platform.

In fact, many transducers based on same quantity transmission principle will always induce that scatter diagrams of their quantity transmission functions present different diagrams because of the tiny difference of geometric structure. For example, for two physical quantities $x$ and $y$ based on same quantity transmission principle, we can respectively plot their scatter diagrams on the $O-x y$ plane through data acquisition, and from the scatter diagram, we can see that because of the different structures of transducers, high linear correlation relationship, or low linear correlation relationship may present between them, and even nothing correlation are presented (seen in (a), (b), (c) of Figure 1).

\subsection{Correlation coefficient method}

The correlation coefficient method is a sort of exact quantitative analysis method in the research of transducer quantity transmission character, and its concrete analysis approaches include taking the acquired transducer input and output data table as the start, taking the measured physical quantity as the independent variable $x$, taking the output as the function $y$, then filling them into the standard deviation list one by one, and finally acquiring the linearization correlation coefficient $r$ corresponding with the transducer by the product moment method in the statistics, and $r$ will directly reflect the linearization degree of the transducer, and the change of structure parameter of every transducer will be denoted by $r$. The meaning of this method is that we can make certain adjustment to the structure parameter of the transducer according to the correlative quantitative analysis between input and output, and convert the transmission function to the linear direction, and offer convenience for the linearization of signal processing of the measurement circuit. After the structure of the transducer is confirmed, the correlation coefficients are also confirmed. When the structure of the transducer is changed, the correlation coefficients are also changed. So we can optimize the correlation coefficients through adjusting the structure parameters of the transducer, and accordingly enhance the linearization degree of the transducer transmission character.

\section{Acquirement of transducer correlation coefficients}

According to the quantity transmission data table of transducer, we can utilize the product moment method or the EXCEL method in statistics to compute the correlation coefficient $r$. Taking the initial parallel flat structure (seen in Figure 3, and the corresponding quantity transmission data table is seen in Figure 1) of the capacitance solid particulate matter moisture transducer as the example, we introduce above two sorts of computation methods of correlation coefficient. 
For the product moment method, first define $x$ as the moisture content $\mathrm{H}(\%)$ of the measured matter, and define $y$ as the output capacitance $\mathrm{Cx}(\mathrm{pF})$ of the capacitance transducer, take the quartz sands with different moisture contents as the measured matters, acquire a series of original data and respectively them into the column $x$ and the column $y$ of the transducer standard deviation table, and then respectively compute corresponding $x-\bar{x}, y-\bar{y}$, $x-\bar{x}, y-\bar{y},(x-\bar{x})^{2},(y-\bar{y})^{2}$ and $(x-\bar{x})(y-\bar{y})$, and fill them in the table one by one (seen in Table 1).

Then, compute corresponding correlation coefficient by the following product moment method formula.

$$
r=\frac{\sigma_{x y}^{2}}{\sigma_{x} \sigma_{y}}
$$

Where,

$$
\sigma_{x y}^{2}=\frac{\sum(x-\bar{x})(y-\bar{y})}{n}
$$

is called as the covariance of independent variable sequence and the attributive variable sequence.

$$
\sigma_{x}=\sqrt{\frac{\sum(x-\bar{x})^{2}}{n}}
$$

is called as the standard deviation of the independent variable sequence.

$$
\sigma_{y}=\sqrt{\frac{\sum(y-\bar{y})^{2}}{n}}
$$

is called as the standard deviation of the attributive variable sequence. So the correlation coefficient formula can be also denoted as

$$
r_{1}=\frac{\sum(x-\bar{x})(y-\bar{y})}{\sqrt{\sum(x-\bar{x})^{2}} \sqrt{\sum(y-\bar{y})^{2}}}=\frac{59.5264}{\sqrt{14.515 \times 245.0291}}=0.97528
$$

Except for the above method, we can also adopt the EXCLE method to acquire $r$. We can utilize the CORREL function to exactly compute the correlation coefficients in Microsoft Office Excel, and the concrete program is

$$
f_{x}=\operatorname{CORREL}\left(A_{1}: A_{n}, B_{1}: B_{m}\right)
$$

Where, $A_{1}$ is the first item of the independent variable sequence, $A_{n}$ is the last item of the sequence, $B_{1}$ is the first item of the attributive variable sequence, and $B_{m}$ is the last item of the corresponding sequence.

Using this method, we can obtain the correlation coefficient of the square taper transducer in Table 1, and the scatter diagram corresponding with this group of data is Figure 2.

Therefore, the scatter diagram of transducer transmission character and the correlation coefficient are two sorts of description of the linearization degree, and the scatter diagram is a sort of diagram method which can not be quantified. But the correlation coefficient is abstract and exact, and it can give exact answer to the difference between result and ideal objective. They describe the transmission characters of the transducer from two different aspects.

\section{Transducer structure parameter adjustment and quantity transmission character improvement}

From the capacitance transducer scatter diagram of above parallel flat structure, we can see that the linearization degree of the quantity transmission character corresponding with this group of data is not high, and the transducer must be improved from the structure, so how to improve the linearization degree through changing the structure of the transducer.

The design principle of most transducers is to confirm the value region range with measurement values between one 
non-electric physical quantity $x$ with another one electric physical quantity $y$ first, and then design corresponding structure according to certain quantity transmission principle existing in these two physical quantities.

In the transducer technology, the linearization degree of the transducer will directly influence the later application of the transducer. The quality of the correlation is reflected by the correlation coefficient, i.e. the correlation coefficient of the transducer is more close to the maximum 1, its linearization degree is higher, and the design aim of the transducer should make its correlation coefficient approach 1 infinitely, thus the burden of the latter measurement circuit will be smaller and the measurement precision of the apparatus will be higher, or else, the transmission function with low linearization degree will increase the difficulty to the linearization processing work for the later measurement circuit, and directly influence the precision class of the measuring apparatus.

Through repetitive researches to the geometric structure of the transducer, we find that in the design of the transducer, the linearization degree of many transducers are always optimized by adjusting their geometric structure parameters, and the linearization degree can be denoted by the correlation coefficient $r$ of the data list of the input and output, and for the ideal linear correlation character of the transmission function, the correlation coefficient should be $100 \%$, i.e. $r=1$. In actual situation, the correlation coefficient is always little smaller than 1 because of various reasons. Obviously, 1 is the upper limit of the actual transducer correlation coefficient, and the difference with 1 is smaller, its linearization is higher. In general economic data statistics, if only the correlation coefficient among data sequences achieves above 0.8 , it thinks high correlation is possessed among data sequences, but in the researches between the structure parameters of the transducer and its linear correlation coefficient, we must use the concept of engineering science to study the problem, and the judgment standards of the correlation are much higher, and for the transducer used in precision measuring apparatus, we divide the correlation coefficient $r$ into three value regions and three corresponding classes, i.e. low correlation, significant correlation and high correlation (seen in Table 2).

Next, taking the capacitance solid particulate matter moisture transducer as the example, we introduce the application of the correlation coefficient method. The initial structure of the transducer is seen in Figure 3, and this is a sort of square taper double-pole capacitance transducer, and in the transmission function, the moisture content of the measured matter is the independent variable, and the electric capacitance of the transducer is the attributive variable. When designing the moisture tester of quartz sand, the moisture content and output capacitance values and the moisture content scatter diagram respectively are seen in $x$ and $y$ in Table 1 and Table 2. From the tables, we can see that though various scatter points of the moisture content and the output capacitance are not in one beeline, but it goes to linearization. Through analyzing the space distribution character of static filed of the square taper capacitance, because the electric field lines have serious aberrances in the marginal region of the pole board, which is the main reason to induce the non-linear transmission character. To improve the linear correlation character, we change the former square taper structure design to circular taper structure of the transducer electric pole board (seen in Figure 4). According to the Euler polygons digital method, we can acquire exact spatial electric field line distribution ((a) and (b) in Figure 4 reflect the fact that the aberrances of the electric field line distribution obvious weaken when the structure of the transducer is changed from square taper to circular taper). Then further increase the radius of the electric pole in the column, and basically control the marginal aberrance of the electric field in the ideal degree (seen in Figure 5 and Table 2).

In the above structure parameter adjustment process of the transducer and the corresponding electric field distribution, we can obviously see that the serious aberrance occurs in the marginal region of the electric field distribution of the square taper transducer parallel pole board, and after the interior and exterior electric poles are changed into cylinder structure and circular arc face structure, the aberrance in the marginal region of the electric filed distribution is obviously improved, and after the exterior electric pole doesn't change and the electric pole radius in the cylinder increase, we acquire ideal electric filed distribution.

The influence induced by the marginal aberrance can be ignored basically. Taking the quartz sand with even granularity as the measured matter, we respective use the capacitance transducers with above three different structures to measure the moisture, and the acquired data are seen in Table 4 and their corresponding scatter diagrams are respectively seen in Figure 6 and Figure 7. Comparing Figure 2 with Figure 6 and Figure 7, we can see that, every time adjustment of transducer structure parameters can improve the linearization degree, and the corresponding 
correlation coefficient is more and more close to the maximum 1. Using the EXCEL-CORREL function method, we can compute the correlation coefficients of three groups of data as follows.

The first group (parallel flat structure): $r_{1}=0.97528$

The second group (small inner core structure): $r_{2}=0.990561$

The third group (big inner core structure): $r_{3}=0.997494$

\section{Conclusions}

Comparing the correlation coefficients of the transducers with three sorts of structure and corresponding scatter diagrams, we can see that the transducer linearization degree with the parallel flat structure belongs to the level of "low correlation", and the transducer linearization degree with the small inner core structure belongs to the level of "significant correlation", and the transducer linearization degree with the big inner core structure belongs to the level of "high correlation", and obviously, the big inner core structure is a sort of ideal structure. Therefore, in the actual measuring works, for the granular solid matter, the square taper transducer has best acceptance nature to the measured matters (such as rapeseed and other croppers), and the second scheme after improvement takes second place, and the third scheme is the worst one, and the designer can carefully balance the advantages and disadvantages to the using object of the transducer.

Obviously, through analyzing the correlation coefficients of the transducer, we can make the structure parameters of the transducers continually approach to the ideal value of the correlation coefficient $(r=1)$, and finally make the quantity transmission character of the transducer achieve ideal level. This method can be generally used in the structure design of the transducer.

\section{References}

Liu, Douren \& Han, Baojun. (2003). Transducer Principle and Application Technology. Xi'an: Xi'an Electrical Science and Technology University Press. P.237-239.

OMRON. (1999). Transducer (Mini Edition). P.772-7512.

Sang, Bingju. (1989). Transducer Transmission Character Curve Fitting. Aero Weaponry Experiment Shooting Range. No.2.

Sun, Baowen \& Yang, Baoqing. (2004). Transducer and Application Manual. Beijing: Mechanical Industry Press. P.223-244.

Yang, Bangwen. (2004). Practical Handbook of New Transducer. Beijing: Renmin Post Press. P.166-273. 
Table 1. Transducer data and standard deviation list

\begin{tabular}{|c|c|c|c|c|c|c|c|c|}
\hline No. & $x$ & $y$ & $x-\bar{x}$ & $(x-\bar{x})^{2}$ & $y-\bar{y}$ & $(y-\bar{y})^{2}$ & $(x-\bar{x})(y-\bar{y})$ & $x y$ \\
\hline 1 & 1.58 & 4.10 & -1.54 & 2.3716 & -7.14 & 50.9796 & 10.9956 & 6.4780 \\
\hline 2 & 1.77 & 5.06 & -1.35 & 1.8225 & -6.18 & 38.1924 & 8.3430 & 8.9562 \\
\hline 3 & 1.92 & 7.01 & -1.20 & 1.4400 & -4.23 & 17.8929 & 5.0760 & 13.4592 \\
\hline 4 & 2.20 & 7.52 & -0.92 & 0.8464 & -3.72 & 13.8384 & 3.4224 & 16.5440 \\
\hline 5 & 2.44 & 9.31 & -0.68 & 0.4624 & -1.93 & 3.7249 & 1.3124 & 22.7164 \\
\hline 6 & 2.74 & 11.50 & -0.65 & 0.4225 & 0.26 & 0.0676 & -0.1690 & 31.5100 \\
\hline 7 & 3.02 & 11.50 & -0.10 & 0.0100 & 0.26 & 0.0676 & -0.0260 & 34.7300 \\
\hline 8 & 3.37 & 11.85 & 0.25 & 0.0625 & 0.61 & 0.3721 & 0.1525 & 39.9345 \\
\hline 9 & 3.71 & 12.73 & 0.59 & 0.3481 & 1.49 & 2.2201 & 0.8791 & 47.2283 \\
\hline 10 & 4.02 & 13.51 & 0.90 & 0.8100 & 2.27 & 5.1529 & 2.0430 & 54.3102 \\
\hline 11 & 4.29 & 17.50 & 1.17 & 0.3689 & 6.26 & 39.1876 & 7.3242 & 75.0750 \\
\hline 12 & 4.64 & 16.82 & 1.52 & 2.3104 & 5.61 & 31.4721 & 8.5272 & 78.0448 \\
\hline 13 & 4.92 & 17.71 & 1.80 & 3.2400 & 6.47 & 41.8609 & 11.6460 & 75.9759 \\
\hline$\sum$ & 40.62 & 146.12 & -0.21 & 14.5153 & 0.03 & 245.0291 & 59.5264 & 504.9625 \\
\hline
\end{tabular}

Note: $\bar{x}=3.12, \bar{y}=11.24$.

Table 2. Correlation class and correlation coefficient value region of capacitance transducer

\begin{tabular}{|c|c|c|c|}
\hline Correlation class & Low correlation & Significant correlation & High correlation \\
\hline$r$ value region & $0.980000 \sim 0.984000$ & $0.986000 \sim 0.992000$ & $0.994000 \sim 0.998000$ \\
\hline
\end{tabular}

Table 3. Parameter adjustment of capacitance transducer structure

\begin{tabular}{|c|c|c|c|c|}
\hline \multirow{2}{*}{ Geometric structure } & \multicolumn{4}{|c|}{ Structural parameters } \\
\cline { 2 - 5 } & $a(\mathrm{~mm})$ & $\phi\left(^{\circ}\right)$ & $R_{1}(\mathrm{~mm})$ & $R_{2}(\mathrm{~mm})$ \\
\hline Before adjustment & 86 & 40 & 6 & 46 \\
\hline After adjustment & 120 & 45 & 11 & 46 \\
\hline
\end{tabular}


Table 4. Moisture content and output capacitance correlation table obtained by square taper transducer when measuring quartz sand samples

\begin{tabular}{|c|c|c|c|c|c|c|}
\hline \multirow[b]{2}{*}{ Test No. } & \multicolumn{2}{|c|}{$\begin{array}{c}\text { First group (parallel flat } \\
\text { structure }\end{array}$} & \multicolumn{2}{|c|}{$\begin{array}{l}\text { Second group (small inner } \\
\text { core structure) }\end{array}$} & \multicolumn{2}{|c|}{$\begin{array}{l}\text { Third group (big inner } \\
\text { core structure) }\end{array}$} \\
\hline & $\begin{array}{l}\text { Moisture } \\
\mathrm{H}(\%)\end{array}$ & $\begin{array}{c}\text { Capacitance } \\
\text { output Cx } \\
(\mathrm{pF})\end{array}$ & $\begin{array}{l}\text { Moisture } \\
\mathrm{H}(\%)\end{array}$ & $\begin{array}{l}\text { Capacitance } \\
\text { output Cx } \\
(\mathrm{pF})\end{array}$ & $\begin{array}{l}\text { Moisture } \\
\mathrm{H}(\%)\end{array}$ & $\begin{array}{c}\text { Capacitance } \\
\text { output Cx } \\
(\mathrm{pF})\end{array}$ \\
\hline 01 & 1.58 & 4.10 & 1.60 & 4.09 & 1.57 & 3.79 \\
\hline 02 & 1.77 & 5.06 & 1.79 & 5.02 & 1.72 & 5.01 \\
\hline 03 & 1.92 & 7.01 & 1.92 & 5.94 & 1.97 & 6.22 \\
\hline 04 & 2.20 & 7.52 & 2.20 & 6.97 & 2.24 & 7.54 \\
\hline 05 & 2.44 & 9.31 & 2.43 & 8.56 & 2.43 & 9.00 \\
\hline 06 & 2.74 & 11.50 & 2.74 & 11.2 & 2.77 & 10.67 \\
\hline 07 & 3.02 & 11.50 & 3.06 & 12.07 & 3.11 & 12.01 \\
\hline 08 & 3.37 & 11.85 & 3.39 & 12.09 & 3.46 & 13.02 \\
\hline 09 & 3.71 & 12.73 & 3.70 & 14.31 & 3.71 & 14.37 \\
\hline 10 & 4.02 & 13.51 & 4.06 & 14.99 & 4.06 & 15.96 \\
\hline 11 & 4.29 & 17.50 & 4.36 & 17.32 & 4.32 & 17.30 \\
\hline 12 & 4.64 & 16.82 & 4.67 & 17.33 & 4.63 & 18.62 \\
\hline 13 & 4.92 & 17.71 & 4.90 & 18.95 & 4.92 & 19.51 \\
\hline $\begin{array}{l}\text { Correlation } \\
\text { coefficients }\end{array}$ & \multicolumn{2}{|c|}{$r_{1}=0.97528$} & \multicolumn{2}{|c|}{$r_{2}=0.990561$} & \multicolumn{2}{|c|}{$r_{3}=0.997494$} \\
\hline
\end{tabular}

Note: Series sample moistures are prepared by the even soakage method.

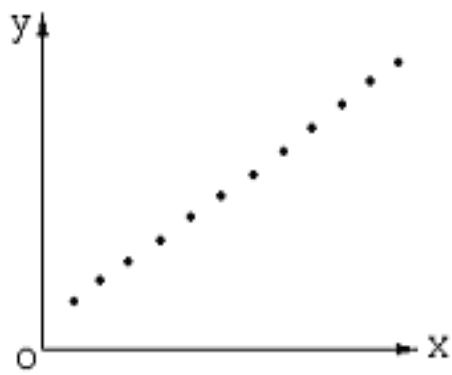

(a) higher linear correlation

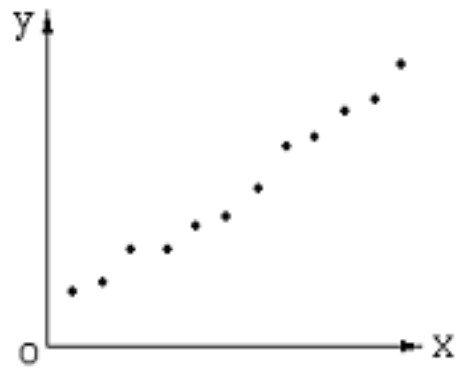

(b) high linear correlation

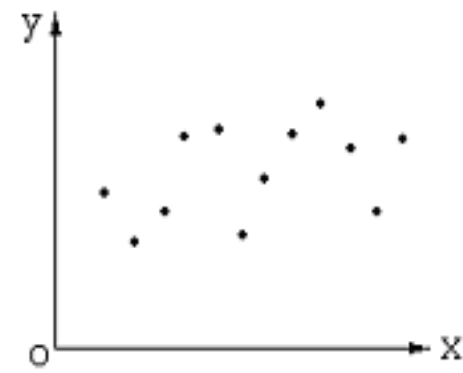

(c) without correlation

Figure 1. Scatter Diagram of Transducer Quantity Transmission Character 


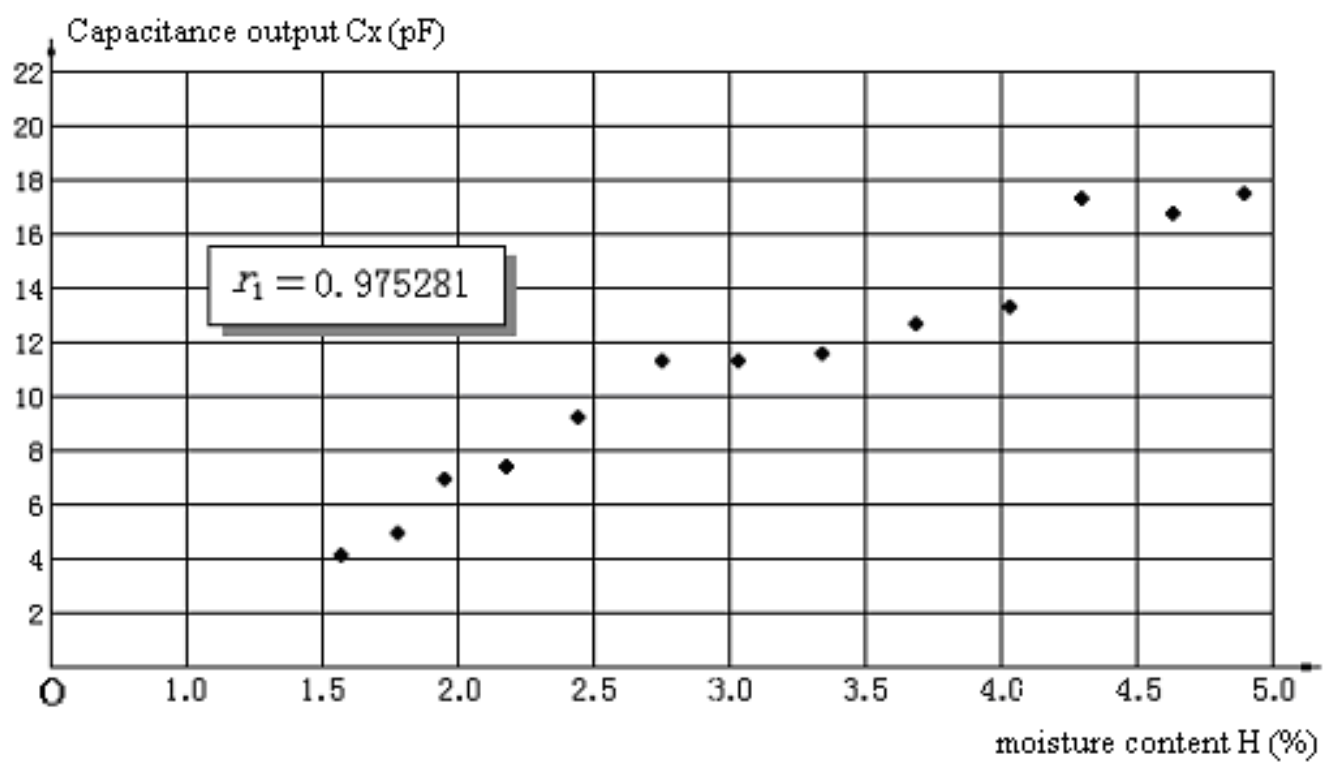

Figure 2. Scatter Diagram of the First Group (Parallel Flat Structural Transducer)

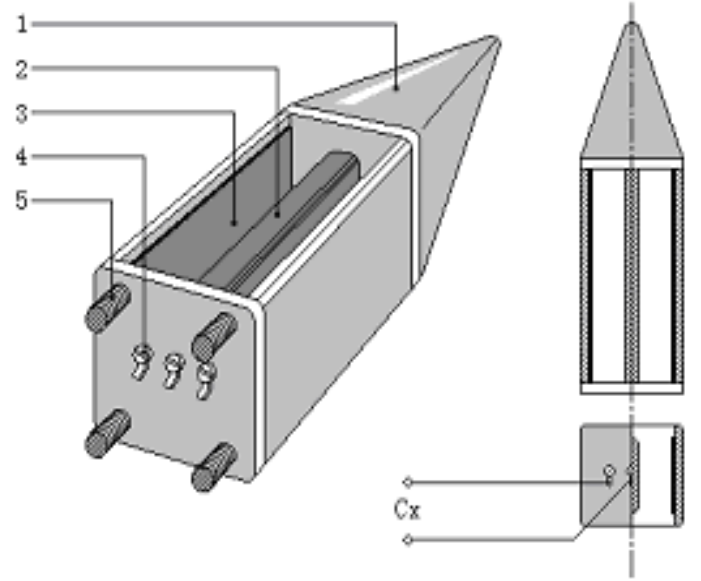

1- Square taper insert detector

2-Center rectangle pole

3- Exterior flat pole

4 Double-pole capacitance pole pin

5 - Fixed bolt

Figure 3. Square Taper Double-pole Capacitance Transducer Structure Sketch (Initial Stage Structure) 


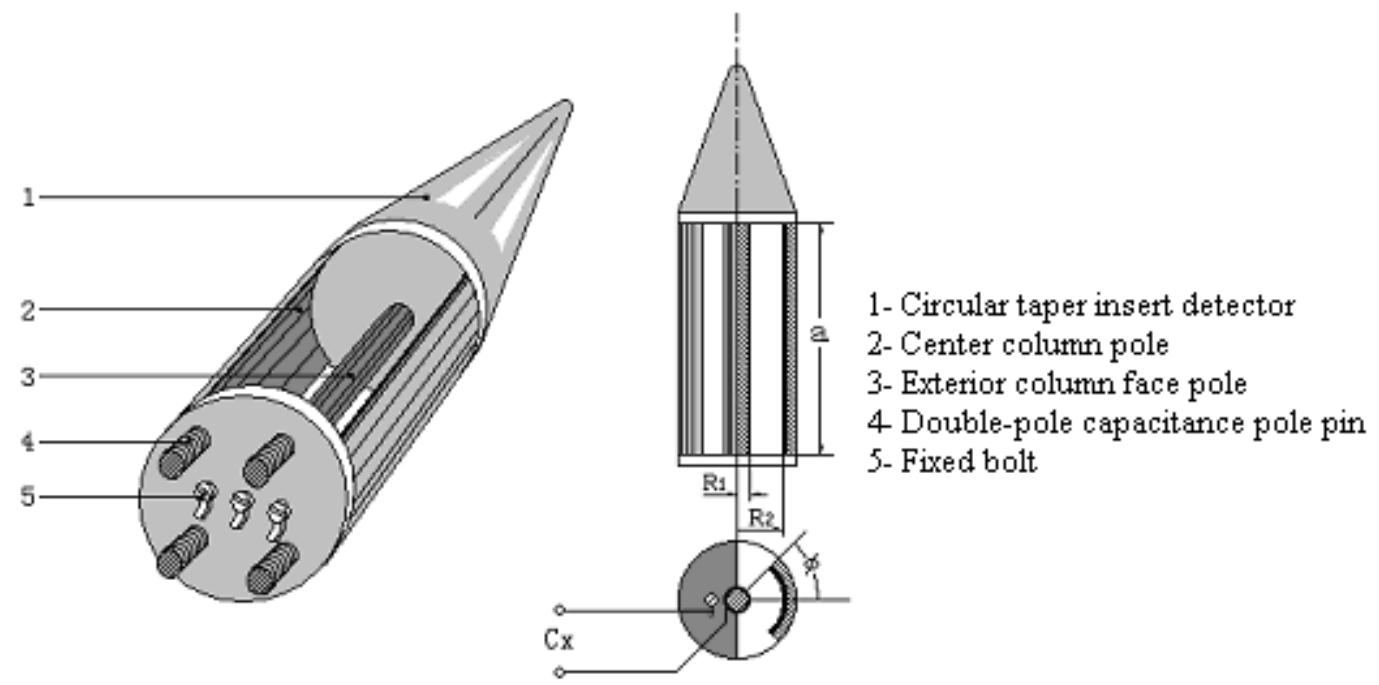

Figure 4. Circular Taper Double-pole Capacitance Transducer Structure Sketch (Improved)

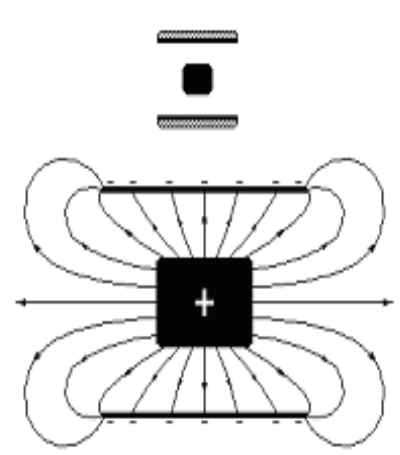

(a) square taper sensor electric field lines (marginal electric field aberrance is serious)

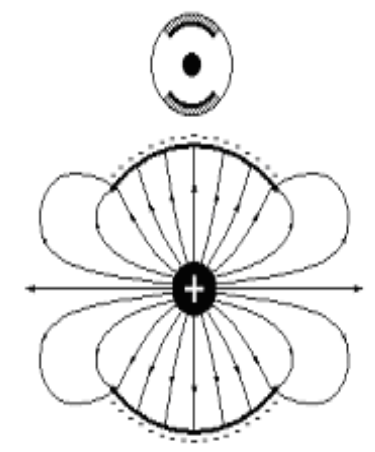

(b) improved taper sensor electric field lines (marginal electric field aberrance is little good)

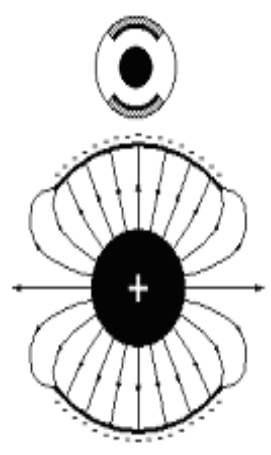

(c) second improved taper sensor electric field lines (marginal electric field aberrance obviously change little)

Figure 5. Different Transducer Structure and Electric Field Aberrance Degree 


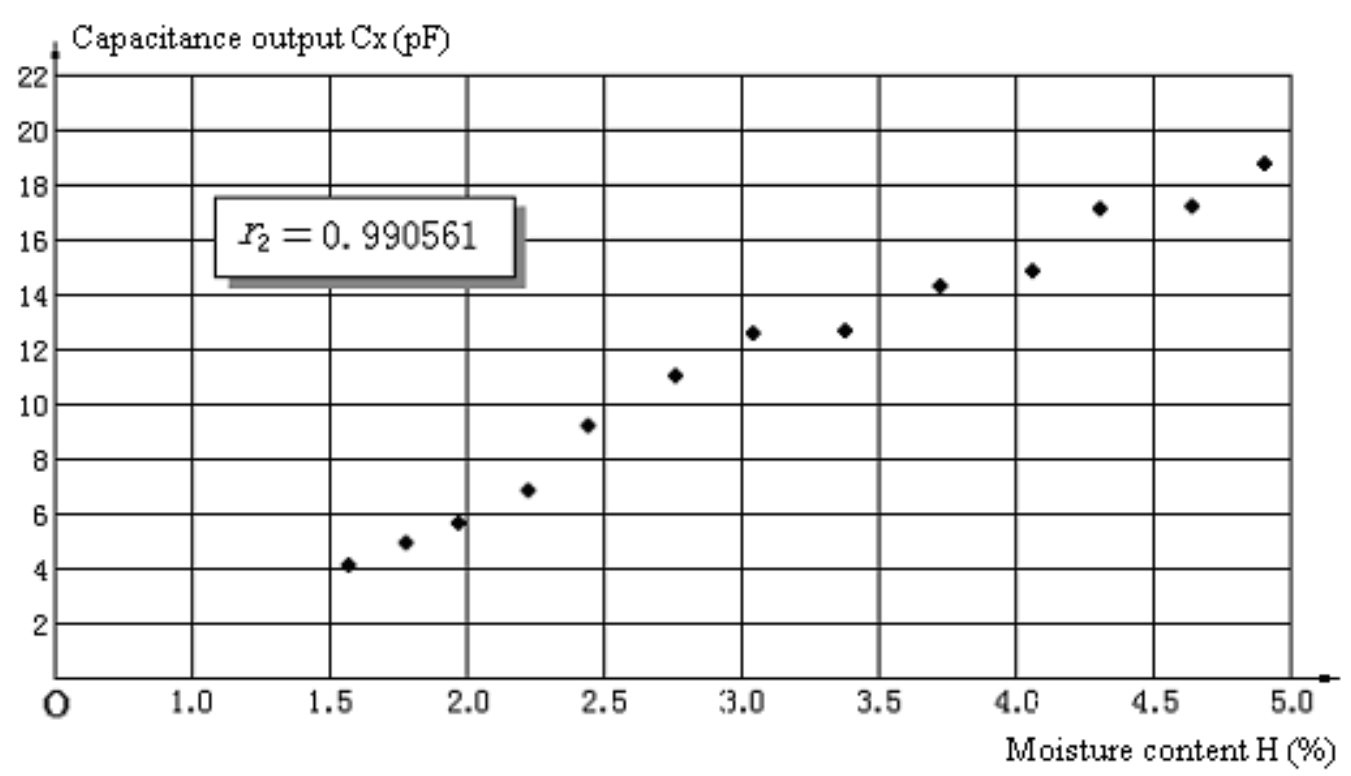

Figure 6. Scatter Diagram of the Second Group (Circular Taper Small Inner Core Capacitance Transducer Structure)

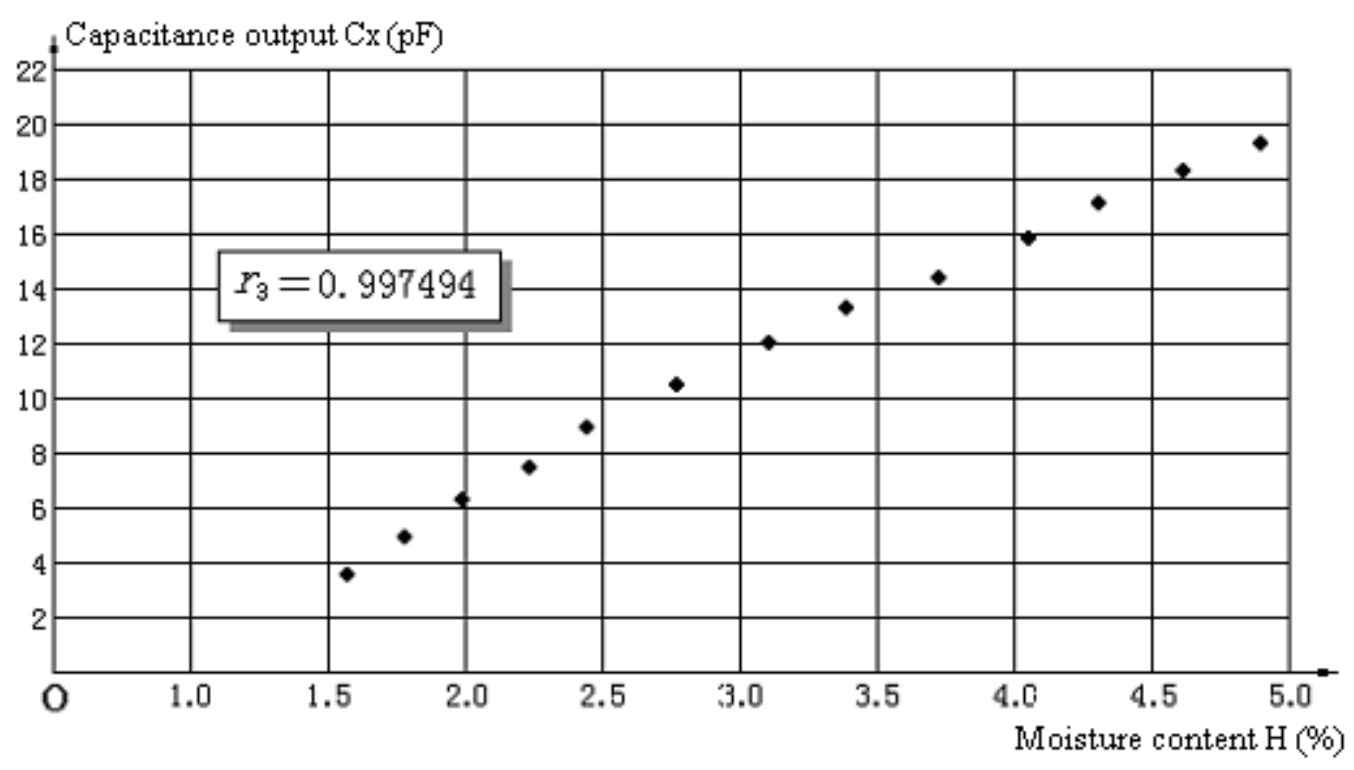

Figure 7. Scatter Diagram of the Third Group (Circular Taper Big Inner Core Capacitance Transducer Structure) 